\title{
DETERMINAÇÃO DE CORANTES MARCADORES DO TIPO AZO E ANTRAQUINONA EM COMBUSTÍVEIS POR CROMATOGRAFIA LÍQUIDA COM DETECÇÃO ELETROQUÍMICA
}

\author{
Magno Aparecido Gonçalves Trindade, Daniel Rinaldo, Wagner Vilegas e Maria Valnice Boldrin Zanoni* \\ Instituto de Química, Universidade Estadual Paulista, CP 355, 14801-970 Araraquara - SP, Brasil
}

Recebido em 23/3/09; aceito em 15/6/09; publicado na web em 27/11/09

\begin{abstract}
DETERMINATION OF AZO AND ANTHRAQUINONE DYES IN FUELS SAMPLE USING HPLC WITH ELECTROCHEMICAL DETECTION. An analytical method based on high-performance liquid chromatography with electrochemical detection has been developed and applied to the determination of Solvent blue 14 (SA-14) and Solvent red 24 (SV-24) in fuel samples. The dyes were better separated on C18 column, using a mobile phase composed of acetonitrile and ammonium acetate (90:10, v/v). Detection was carried out at an oxidation potential of $+0.85 \mathrm{~V}$. The detector response was linear at concentration range of $7.50 \times 10^{-8}-1.50 \times 10^{-6} \mathrm{~mol} \mathrm{~L}^{-1}(\mathrm{r}=0.997)$ for SA-14 and SV-24, respectively. The method was used to quantify these dyes in fuels samples with satisfactory accuracy and precision.
\end{abstract}

Keywords: solvent Blue 14 dye; solvent Red 24 dye; fuel analysis.

\section{INTRODUÇÃO}

As diferenças de tratamento fiscais nas utilizações de combustíveis derivados do petróleo nos diversos segmentos - tais como, pesca, mineração, geração de energia elétrica, aquecimento de residências, agricultura, transportes e lazer - deram origem a um imenso mercado de corantes como marcadores destes produtos. Atualmente, este sistema de marcação de combustíveis tem sido usado para prevenir fraudes fiscais como, por exemplo, em países da África que lutam contra o contrabando de derivados de petróleo, até países da União Europeia e Estados Unidos, que propiciam tratamento fiscal diferenciado a produtos com finalidades diferentes como, por exemplo, óleo diesel usado para aquecimento de residências, tráfego de rodovias e uso em equipamentos agrícolas, gasolina com ou sem chumbo, combustível com ou sem subsídio etc. ${ }^{1-6}$

O uso de corantes na indústria de combustíveis usualmente não está relacionado ao apelo estético ou melhoramento na performance do combustível, porém, são particularmente usados para identificar tipos específicos de combustíveis, visando auxiliar o processo de controle e fiscalização do fabricante ou das instituições responsáveis pela procedência e/ou destino do combustível gerado. ${ }^{7}$

Os corantes do tipo solvente azul 14 (SA-14) e solvente vermelho 24 (SV-24) (Figura 1, inserção) não são solúveis em água, apresentam grupos antraquinona e azo, ${ }^{8,9}$ respectivamente, como cromóforo e, são usualmente empregados como marcadores visíveis em diversos tipos de combustíveis. Devido ao grande número de corantes marcadores, com similaridade de coloração disponíveis comercialmente com diversidades de estruturas, bem como propriedades físico-químicas aliadas à complexidade das matrizes oriunda de combustíveis derivados do petróleo, os métodos analíticos capazes de se adequar a estas diversidades são restritos. De acordo com isso, a técnica de cromatografia líquida de alta eficiência (CLAE) acoplada a diversos detectores, tais como absorção no ultravioleta/visível e arranjo de diodos, ${ }^{10-13}$ espectrometria de massa ${ }^{14}$ e espectroscopia derivativa ${ }^{15}$ tem sido uma das técnicas analíticas mais utilizadas na identificação e quantificação destes marcadores. Recentemente, a aplicação de métodos eletroquímicos na quantificação dos corantes quinizarina ${ }^{16} \mathrm{e}$ solvente azul $14^{17} \mathrm{em}$ amostras de combustíveis tem sido investigada pelo nosso grupo de trabalho e resultados satisfatórios foram obtidos

\footnotetext{
*e-mail: boldrinv@iq.unesp.br
}

para quantificação destes corantes quando presentes separadamente na amostra. Ainda que a técnica cromatográfica com detecção eletroquímica seja explorada no desenvolvimento de métodos analíticos para a quantificação de uma grande variedade de analitos orgânicos devido a sua alta detectibilidade, nenhum trabalho tem sido reportado na literatura para quantificação de corantes quando presentes na forma combinada em amostras de combustíveis.

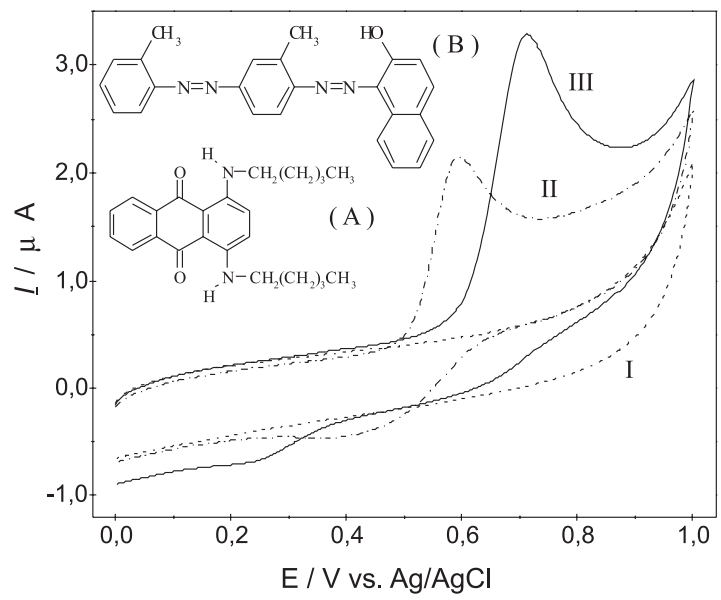

Figura 1. Voltamograma cíclico registrado sobre eletrodo de carbono vítreo para: (I) branco e oxidação de $1,00 \times 10^{-4} \mathrm{~mol} \mathrm{~L}^{-1}$ dos corantes (II) SA-14 e (III) SV-24 em meio de ACN/acetato de amônio 0,10 mol L-1 (70:30, $v / v)$. Inserção: Fórmula estrutural dos corantes (A) SA-14 e (B) $S V-24$. $v=100 \mathrm{mV} \mathrm{s}^{-1}$

Portanto, foi objetivo do presente trabalho investigar a possibilidade de análise simultânea dos corantes SA-14 e SV-24 em amostras de combustíveis utilizando CLAE acoplado a um detector eletroquímico.

\section{PARTE EXPERIMENTAL}

\section{Reagentes, solventes e soluções}

Os corantes SA-14 (Acros Organics, EUA, 2005) e SV-24 (Aldrich, EUA, 2005), grau analítico, foram preparados como solução estoque de 1,00 $\mathrm{mmol} \mathrm{L}^{-1}$ em acetonitrila (grau CLAE, J. T. Baker) 
e usados após diluições nas concentrações desejadas. Cloreto de lítio (Mallinckrodt), acetato de amônio (Merck), ácido fórmico (Merck) e ácido acético (Merck), todos de grau de pureza analítica, utilizados como eletrólito suporte foram preparados em água purificada com sistema de Milli- $Q$ (Millipore, modelo Simplicity 185). Os solventes metanol (J. T. Baker) e acetonitrila (J. T. Baker) de grau CLAE foram utilizados como fase móvel para separação dos corantes, hexano (J. T. Baker) de grau CLAE foi usado durante o procedimento de extração.

\section{Amostras de combustíveis}

As amostras de combustíveis, querosene (JET A1) e etanol combustível, certificadas da isenção dos corantes estudados, foram coletadas na cidade de Araraquara/SP-Brasil e deixadas sob refrigeração. Soluções dos padrões dos corantes de interesse foram preparadas e, a seguir, intencionalmente adicionadas nas amostras para dar a concentração desejada de cada corante nessas amostras.

\section{Equipamentos}

As análises cromatográficas foram realizadas em um sistema de cromatografia líquida de alta eficiência (CLAE) (Varian ProStar, modelo 210) equipado com os detectores de espectrofotometria na região do UV/Vis (Varian ProStar, modelo 320) e eletroquímico (Varian ProStar, modelo 370) operando em modo amperométrico. No sistema utilizou-se uma célula eletroquímica do tipo wall-jet constituída de três eletrodos, carbono vítreo com área geométrica de 0,71 $\mathrm{cm}^{2}$ (eletrodo de trabalho), um disco de platina (eletrodo auxiliar) e $\mathrm{Ag} / \mathrm{AgCl}$ (eletrodo de referência). Um cromatógrafo líquido de alta eficiência acoplado a um detector de arranjo de fotodiodos (CLAEDAD) da marca Jasco ${ }^{\circledR}$ (bomba modelo PU-2089, DAD MD-2010, DC CD-2095, injetor automático AS-2055) foi usado para obter resultados comparativos. A separação dos corantes foi otimizada em coluna analítica de fase reversa Phenomenex ${ }^{\circledR}$ Luna C-18 (250 x 4,60 $\mathrm{mm}, 5,00 \mu \mathrm{m}$ ), a qual foi protegida com uma pré-coluna (Security Guard Holder, Phenomenex ${ }^{\circledR}$ ). Ultrassom (Unique) foi utilizado para homogeneização das soluções e para degaseificação dos solventes da fase móvel.

As medidas eletroquímicas foram realizadas utilizando-se um potenciostato/ galvanostato (Autolab, modelo PGSTAT 30), interfaceado a um microcomputador e gerenciado pelo software GPES 4.9 (Eco Chemie) para aquisição dos dados. Foi utilizado um sistema convencional de três eletrodos composto por um eletrodo de disco de carbono vítreo (Metrohm, diâmetro de 2,00 mm) como eletrodo trabalho, um eletrodo de $\mathrm{Ag} / \mathrm{AgCl}_{\text {sat }}$ (inserido em um capilar de $\mathrm{Lu}$ ggin contendo $\mathrm{KCl}$ 3,00 $\mathrm{mol} \mathrm{L}^{-1}$ ), como eletrodo de referência, e um fio de platina usado como eletrodo auxiliar. Para este sistema, uma célula convencional com capacidade máxima de $10,0 \mathrm{~mL}$ foi utilizada. Antes de cada medida experimental, o eletrodo de carbono vítreo foi polido com solução aquosa de alumina 1,00 $\mu \mathrm{m}$ e, em seguida, enxaguado exaustivamente com água deionizada.

\section{Procedimento para obtenção dos cromatogramas por CLAE-DE e CLAE-DAD}

Inicialmente, o eletrodo de carbono vítreo (célula wall-jet) usado no detector eletroquímico foi polido com pasta de diamante $(0,50 \mu \mathrm{m}$, Varian) sobre um tecido especial adaptado a uma placa de vidro apropriada e enxaguado exaustivamente com água deionizada e acetona. Antes das análises a coluna cromatográfica foi equilibrada com metanol, seguido de acetonitrila $(\mathrm{ACN})$ por aproximadamente $1 \mathrm{~h}$ usando vazão de 0,80 $\mathrm{mL} \min ^{-1}$. Em seguida, o sistema cromatográfico foi estabilizado pela passagem da fase móvel na proporção e vazão requerida para as análi- ses. Após a estabilização, as soluções contendo a mistura dos corantes foram filtradas em membranas filtrante $0,45 \mu \mathrm{m}$ (Millipore) e injetadas manualmente em loop (Varian ProStar) com capacidade para $20 \mu \mathrm{L}$.

Para as análises realizadas por CLAE-DAD utilizou-se um sistema binário composto por solvente $\mathrm{A}\left(\mathrm{H}_{2} \mathrm{O}+0,05 \%\right.$ de ácido trifluoroacético, TFA) e solvente $\mathrm{B}$ ( $\mathrm{ACN}$ ) como melhor condição de fase móvel, cuja percentagem foi mantida em $95,0 \%$ de $\mathrm{ACN}$ e $5,00 \%$ do $\left(\mathrm{H}_{2} \mathrm{O}+0,05 \%\right.$ TFA) sob vazão de $1,70 \mathrm{~mL} \mathrm{~min}^{-1}$. O sinal cromatográfico foi monitorado no intervalo de 200 a $700 \mathrm{~nm}$ e cromatogramas no comprimento de onda mais sensível, $490 \mathrm{~nm}$ (SV-24) e $640 \mathrm{~nm}$ (SA-14), foram selecionados e os parâmetros cromatográficos dos mesmos foram extraídos.

\section{Análise dos corantes SA-14 e SV-24 em amostras de combustíveis}

Nas amostras de álcool comercial (coletadas em postos de abastecimento de Araraquara), foram propositalmente adicionados os corantes em estudo na concentração de 0,$08 ; 0,20$ e 2,00 mg L-1 e injetadas diretamente após serem filtradas em membranas filtrante $0,45 \mu \mathrm{m}$. Diluição prévia foi realizada somente para amostra contendo $2,00 \mathrm{mg} \mathrm{L}^{-1}$ dos corantes, onde $1,00 \mathrm{~mL}$ da amostra foi coletada em balão volumétrico de $5,00 \mathrm{~mL}$ e o volume ajustado com $\mathrm{ACN}$.

Para as amostras comerciais de querosene de aviação "JET A1", foi realizado um procedimento de extração envolvendo sílica ( $\mathrm{Si}$ ). Inicialmente, o material sorvente sílica gel 60 (tamanho de partícula 0,06-0,20 mm, 70-230 mesh ASTM, Bakerbond, J. T. Baker) foi submetido à secagem por, aproximadamente, $4 \mathrm{~h}$ em estufa sob temperatura de $110{ }^{\circ} \mathrm{C}$. A seguir, pesou-se $1000 \mathrm{mg}$ do material seco e adicionou-se em cartucho de extração em fase sólida (EFS) convencional, tipo seringa. O material foi firmemente empacotado utilizando-se um bastão de vidro com diâmetro semelhante ao do cartucho e utilizado como descrito a seguir.

Alíquotas de 3,00 e 1,00 mL de amostra de querosene de aviação "JET A1" contendo, respectivamente, 0,40 e 2,00 $\mathrm{mg} \mathrm{L}^{-1}$ dos corantes SA-14 e SV-24 foram percoladas em cartucho EFS contendo 1000 $\mathrm{mg}$ de $\mathrm{Si}$, previamente condicionado com $5,00 \mathrm{~mL}$ de hexano (grau P.A., QHemis). Após esta etapa, o cartucho foi lavado com aproximadamente $10,0 \mathrm{~mL}$ de hexano e, logo a seguir, o analito foi eluído em balão volumétrico de 5,00 mL com ACN. A mistura resultante foi analisada e a quantificação dos corantes na amostra foi realizada pelo método do padrão externo.

\section{RESULTADOS E DISCUSSÃO}

\section{Otimização dos parâmetros cromatográficos}

Inicialmente, voltamogramas cíclicos em modo estático foram obtidos para oxidação de $1,00 \times 10^{-4} \mathrm{~mol} \mathrm{~L}^{-1}$ dos corantes SA-14 e SV-24 em meio de acetonitrila (ACN) contendo 30,0\% de solução aquosa de acetato de amônio $\left(0,10 \mathrm{~mol} \mathrm{~L}^{-1}\right)$, objetivando conhecer o comportamento voltamétrico desses corantes em condição de eletrólito suporte similar àquela adotada nas análises cromatográficas. De acordo com a Figura 1, observa-se que nesta condição ambos os corantes apresentam picos de oxidação bem definidos em potenciais de, aproximadamente, $+0,59 \mathrm{e}+0,71 \mathrm{~V}$ vs. $\mathrm{Ag} / \mathrm{AgCl}$, respectivamente.

Assim, foram conduzidos os estudos preliminares visando a otimização da melhor condição experimental de análise, na qual se variou a composição da fase móvel, testando-se diferentes combinações e proporções de solventes, tais como, mistura de acetonitrila $(\mathrm{ACN})$ e metanol com cloreto de lítio $\left(1,00 \mathrm{mmol} \mathrm{L}^{-1}\right)$, acetato de amônio $(1,00$ mmol L-1) e ácido acético $\left(1,00 \mathrm{mmol} \mathrm{L}^{-1}\right)$ adotando um potencial de oxidação de $+0,80 \mathrm{~V}$ vs. $\mathrm{Ag} / \mathrm{AgCl}$, cujo potencial foi suficiente para detecção de ambos os corantes na mistura. 
Tabela 1. Parâmetros cromatográficos obtidos para eluição dos corantes SA-14 e SV-24 em fase móvel ACN/acetato de amônia (5,00 mmol L-1) em diferentes proporções de fase móvel. Vazão de $1,70 \mathrm{~mL} \mathrm{~min}^{-1}$ e detecção em $+0,80 \mathrm{~V}$ vs. $\mathrm{Ag} / \mathrm{AgCl}$

Composição da fase móvel ACN/acetato de amônia

$85: 15$

$88: 12$

90:10

\begin{tabular}{lccccccccc}
\hline Picos & $\mathrm{t}_{\mathrm{R}}(\min )$ & $\mathrm{K}^{\prime}$ & $\mathrm{R}_{\mathrm{s}}$ & $\mathrm{t}_{\mathrm{R}}(\min )$ & $\mathrm{K}^{\prime}$ & $\mathrm{R}_{\mathrm{s}}$ & $\mathrm{t}_{\mathrm{R}}(\min )$ & $\mathrm{K}^{\prime}$ & $\mathrm{R}_{\mathrm{s}}$ \\
$\mathrm{SA-14}$ & 4,87 & 3,88 & 6,50 & 4,43 & 3,43 & 5,50 & 4,12 & 3,13 & 4,00 \\
$\mathrm{SV}-24$ & 26,0 & 25,0 & 24,0 & 20,0 & 19,0 & 19,0 & 16,5 & 15,5 \\
\hline
\end{tabular}

$\mathrm{t}_{\mathrm{R}}=$ tempo de retenção; $\mathrm{K}^{\prime}$ = fator de retenção e $\mathrm{R}_{\mathrm{s}}=$ resolução, obtido em relação aos picos mais próximos.

Estudou-se ainda a vazão da fase móvel (entre 1,00 e 1,80 $\mathrm{mL} \min ^{-1}$ ), a concentração do eletrólito de suporte (entre 1,00 e $5,00 \mathrm{mmol} \mathrm{L}^{-1}$ ) e o potencial de detecção (entre $+0,65 \mathrm{e}+1,10$ $\mathrm{V}$ vs. $\mathrm{Ag} / \mathrm{AgCl}$ ), cujos resultados em termos de simetria de pico e tempo de análise obtidos calculando-se os parâmetros cromatográficos são apresentados na Tabela 1. Os melhores resultados foram obtidos usando-se fase móvel ACN/acetato de amônio 5,00 mmol L ${ }^{-1}$ na proporção 90:10 (v/v), vazão $1,70 \mathrm{~mL} \mathrm{~min}^{-1} \mathrm{e}$ detecção em $+0,85 \mathrm{~V}$ vs. $\mathrm{Ag} / \mathrm{AgCl}$. A Figura 2 mostra o perfil cromatográfico correspondente à separação de uma solução contendo $3,00 \times 10^{-6} \mathrm{~mol} \mathrm{~L}^{-1}$ dos corantes SA-14 e SV-24, empregando-se a melhor condição de análise.

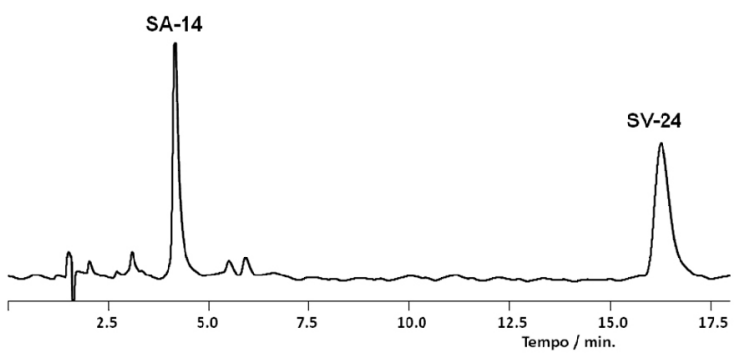

Figura 2. Cromatograma registrado para detecção de 3,00× $10^{-6} \mathrm{~mol} \mathrm{~L}^{-1}$ dos corantes SA-14 e SV-24 eluídos em fase móvel ACN/acetato de amônia (5,00 mmol $\left.\mathrm{L}^{-1}\right)$ na proporção $(90: 10, \mathrm{v} / \mathrm{v})$ sob vazão de $1,70 \mathrm{~mL} \mathrm{~min} \mathrm{~m}^{-1}$ e detecção em $+0,85 \mathrm{~V} v \mathrm{~s}$. Ag/AgCl. Picos: $S A-14 t_{R}=4,20$ min e $S V-24 t_{R}=16,50 \mathrm{~min}$ (unidade eixo $Y, m V$ )

\section{Efeito do potencial na detecção eletroquímica}

O perfil cromatográfico de cada corante foi monitorado medindose a resposta do detector eletroquímico (área do pico) sob aplicação de diferentes voltagens $\left(\mathrm{E}_{\text {detecção }}\right)$ variando-se entre $+0,65 \mathrm{a}+1,10 \mathrm{~V}$ vs. $\mathrm{Ag} /$ $\mathrm{AgCl}$. A Figura 3 mostra os voltamogramas hidrodinâmicos obtidos para a eletro-oxidação de ambos corantes, nos quais pode ser observado que valores ótimos de potencial são obtidos abaixo de $+0,95 \mathrm{~V}$ vs. Ag/ $\mathrm{AgCl}$ para o (SA-14) e acima de $+0,80 \mathrm{~V}$ vs. $\mathrm{Ag} / \mathrm{AgCl}$ para o (SV-24). Em valores de potencial acima de $+0,90 \mathrm{~V}$ vs. $\mathrm{Ag} / \mathrm{AgCl}$ o sinal diminui significativamente, sendo acompanhado de um intenso aumento da corrente de fundo (corrente da célula), levando a um aumento no tempo de estabilização do sistema. Portanto, a escolha do melhor potencial para detecção dos corantes foi um compromisso entre intensidade de sinal e tempo de estabilização da linha-base, o qual analisando a Figura 3 evidencia como valor ótimo o potencial de $+0,85 \mathrm{~V}$ vs. $\mathrm{Ag} / \mathrm{AgCl}$.

\section{Curva analítica e aplicação do método}

Uma curva analítica foi obtida (medindo-se área do pico) empregando-se a melhor condição de trabalho definida como: fase móvel ACN/ acetato de amônia $\left(5,00 \mathrm{mmol} \mathrm{L}^{-1}\right)$ na proporção de $(90: 10 \mathrm{v} / \mathrm{v})$ sob vazão de $1,70 \mathrm{~mL} \mathrm{~min}^{-1}$ e detecção em $+0,85 \mathrm{~V}$ vs. $\mathrm{Ag} / \mathrm{AgCl}$, a qual apresentou

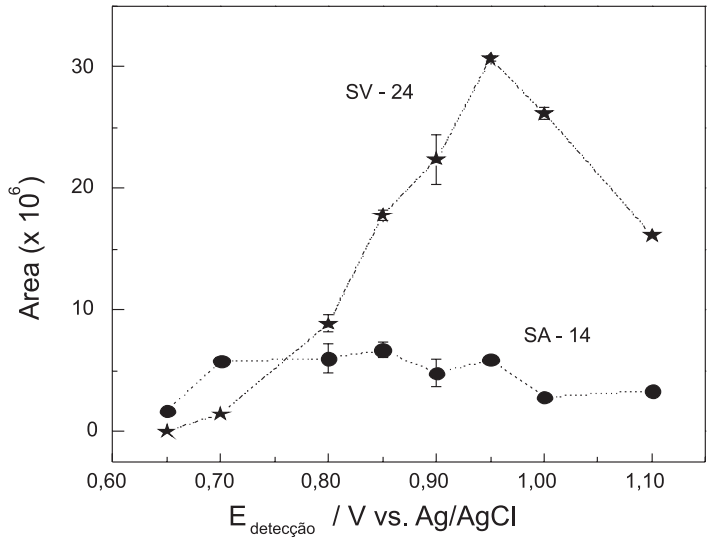

Figura 3. Voltamogramas hidrodinâmicos obtidos para oxidação eletroquímica dos corantes SA-14 $\left(1,00 \xi 10^{-6} \mathrm{~mol} \mathrm{~L}^{-1}\right)$ e SV-24 $\left(4,00 \times 10^{-6} \mathrm{~mol} \mathrm{~L}^{-1}\right)$. Condições experimentais especificadas na Figura 2

resposta linear no intervalo de concentração de $7,50 \times 10^{-8}$ a $1,50 \times 10^{-6}$ mol L-1 (Figura 4) para ambos os corantes estudados, cujas equações de regressão linear podem ser obtidas a partir dos valores dos parâmetros apresentados na Tabela 2. A partir dos parâmetros analíticos extraídos das curvas e reunidos na Tabela 2, estimou-se ainda o limite de detecção (LD) usando a seguinte relação matemática: $(3 \times \mathrm{SD} / b),{ }^{18}$ em que SD representa a estimativa do erro relativo ao coeficiente linear e $b$ a inclinação da curva de calibração. O LD obtido para os corantes SA-14 e SV-24 revela que o sistema proposto apresenta melhor detectibilidade quando comparado com aqueles obtidos por cromatografia com detecção em arranjo de diodo (Tabela 2), de modo que pode ser aplicado para quantificação destes corantes em níveis de concentrações significativamente inferiores.

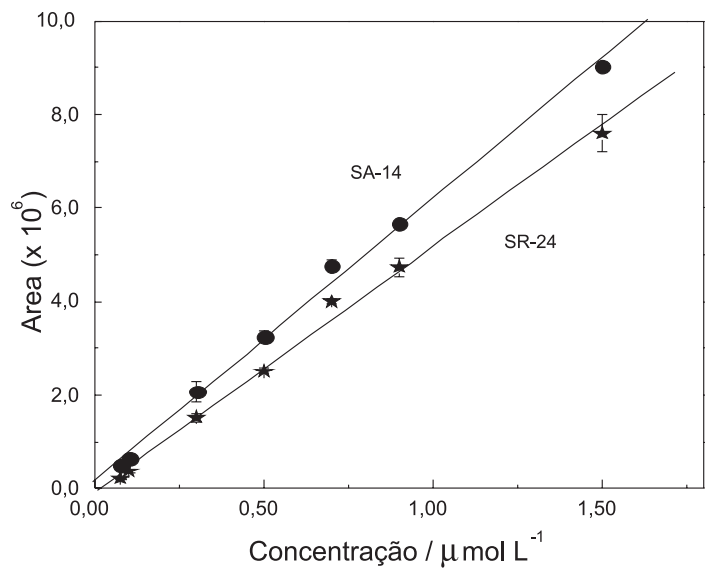

Figura 4. Curva analítica obtida para diferentes concentrações dos corantes SA-14 e SV-24, eluídos em fase móvel ACN/acetato de amônia $\left(5,00 \mathrm{mmol} \mathrm{L}^{-1}\right)$ na proporção de $(90: 10 \mathrm{v} / \mathrm{v})$. Vazão da fase móvel 1,70 $\mathrm{mL} \mathrm{min}{ }^{-1}$ e detecção em $+0,85 \mathrm{~V} v \mathrm{~s} . \mathrm{Ag} / \mathrm{AgCl}$ 
Tabela 2. Comparação dos parâmetros analíticos obtidos pela aplicação do método utilizando CLAE com detecção eletroquímica (CLAE-DE) e por arranjos de fotodiodos (CLAE-DAD)

\begin{tabular}{lcccc}
\hline & \multicolumn{2}{c}{ CLAE-DE } & \multicolumn{2}{c}{ CLAE-DAD } \\
\hline Parâmetros & SA-14 & SV-24 & SA-14 & SV-24 \\
Linearidade $\left(\mathrm{mol} \mathrm{L}^{-1}\right)$ & $7,50 \times 10^{-8} \mathrm{a} 1,50 \times 10^{-6}$ & $7,50 \times 10^{-8} \mathrm{a} 1,50 \times 10^{-6}$ & $(5,00-50,0) \times 10^{-6}$ & $(5,00-50,0) \times 10^{-6}$ \\
Intercepto (mV) & 198727 & -53212 & 617 & -6506 \\
Inclinação (mV L mol $\left.{ }^{-1}\right)$ & $6,02 \times 10^{12}$ & $5,23 \times 10^{12}$ & $9,74 \times 10^{9}$ & $2,80 \times 10^{10}$ \\
r & 0,998 & 0,997 & 0,999 & 0,999 \\
LD (mol L-1) & $6,40 \times 10^{-8}$ & $7,40 \times 10^{-8}$ & $8,00 \times 10^{-7}$ & $1,00 \times 10^{-6}$ \\
\hline
\end{tabular}

$\mathrm{r}=$ Coeficiente de correlação

O método proposto foi aplicado para quantificação dos corantes em amostras de combustíveis (Figura 5). O registro de cromatogramas para análises de amostras de álcool contendo 0,40 e 2,00 $\mathrm{mg} \mathrm{L}^{-1}$ após adição do padrão dos corantes mostrou que não há picos (Figura 5B e C) que possam suprimir a detecção precisa dos corantes em análise e, deste modo, foram realizados estudos de recuperação pelo método proposto. Os resultados obtidos foram satisfatórios com níveis de recuperação aceitáveis, principalmente para o SA-14, cujos valores e tratamento estatístico estão mos-

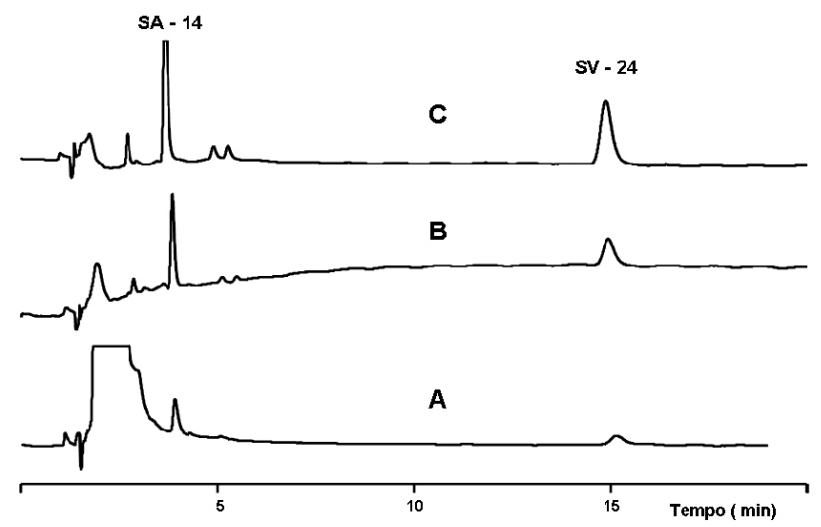

Figura 5. Cromatogramas obtidos para: (A) análise de amostra de querosene contendo $0,40 \mathrm{mg} \mathrm{L}^{-1}$ de $S A-14$ e $S V$-24 após extração usando o procedimento experimental, (B) amostra de álcool contendo $0,40 \mathrm{mg} \mathrm{L}^{-1}$ de SA-14 e SV-24 e (C) amostra de álcool contendo 2,00 mg L $\mathrm{L}^{-1}$ de SA-14 e $S V$-24 obtidos empregando a melhor condição de trabalho como mostrado na Figura 4 (unidade eixo $Y, m V$ ) trados na Tabela 3. A exatidão foi estimada usando-se valores de concentração determinados experimentalmente (recuperação) comparados com a concentração teórica (valor adicionado), como pode ser observado na Tabela 3.

Cromatogramas obtidos para análise de amostra de querosene fortificada com 0,40 $\mathrm{mg} \mathrm{L}^{-1}$ dos corantes SA-14 e SV-24 (Figura 5A) após extração usando o procedimento experimental (2.4), mostra que a detecção dos picos dos corantes não é afetada e nenhuma espécie interferente é coeluída nessas condições de análise. Entretanto, foi obtida recuperação aceitável para a detecção do corante SV-24 somente para as amostras de álcool, cujas análises foram realizadas diretamente, sem a necessidade de pré-tratamento. As recuperações obtidas para análise do corante SV-24 em amostras de querosene foram inferiores a 70,0\% (concentração de $0,40 \mathrm{mg} \mathrm{L}^{-1}$ ) e $50,0 \%$ (concentração de 2,00 $\mathrm{mg} \mathrm{L}^{-1}$ ), o que pode ser justificado pela perda do analito durante o processo de extração (limpeza da amostra com hexano) em função de sua baixa polaridade e, assim, fraca interação com o material sorvente. Isto evidencia que, nestas condições de trabalho, o método proposto pode ser aplicado com sucesso apenas na quantificação do corante SA-14.

Para análises dos corantes SA-14 e SV-24 por CLAE-DAD, otimizaram-se os parâmetros cromatográficos, fator de retenção (K'), seletividade $(\alpha)$ e resolução $\left(R_{s}\right)$, pela variação da composição e percentagem da fase móvel, vazão da fase móvel e escolha do melhor comprimento de onda $(\lambda)$. A melhor condição de análise foi obtida com fase móvel $\mathrm{ACN} / \mathrm{H}_{2} \mathrm{O}$ na proporção de 95:05 (v/v) sob

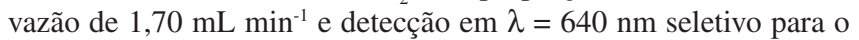
SA-14 e $\lambda=490 \mathrm{~nm}$ seletivo para o SV-24, cuja condição permitiu obter cromatogramas em um tempo de análise aceitável e, assim, esta condição foi adotada para fins analíticos.

Tabela 3. Resultados de recuperação obtidos para quantificação dos corantes SA-14 e SV-24 em amostras de combustíveis por CLAE-DE

\begin{tabular}{|c|c|c|c|c|c|}
\hline Analitos & Matriz & $\begin{array}{c}\text { Adicionado } \\
\left(\mathrm{mg} \mathrm{L}^{-1}\right)\end{array}$ & $\begin{array}{c}\text { Encontrado } \\
\left(\mathrm{mg} \mathrm{L}^{-1}\right) \pm t \cdot \mathrm{DP} / \mathrm{n}^{1 / 2 \mathrm{c}}\end{array}$ & $\begin{array}{c}\text { Recuperado } \\
(\%)\end{array}$ & $\begin{array}{c}\text { DPR } \\
(\%) \\
\end{array}$ \\
\hline \multirow{5}{*}{ SA-14 } & Álcool ${ }^{\mathrm{a}}$ & 0,08 & $0,07 \pm 0,01$ & 87,5 & 2,70 \\
\hline & & 0,20 & $0,17 \pm 0,01$ & 84,7 & 4,00 \\
\hline & & 2,00 & $1,75 \pm 0,02$ & 87,8 & 4,00 \\
\hline & Querosene ${ }^{\mathrm{b}}$ & 0,40 & $0,30 \pm 0,03$ & 75,0 & 5,20 \\
\hline & & 2,00 & $1,45 \pm 0,16$ & 72,5 & 6,00 \\
\hline \multirow{5}{*}{ SV-24 } & Álco ${ }^{a}$ & 0,08 & $0,06 \pm 0,02$ & 78,4 & 10,0 \\
\hline & Alcool ${ }^{4}$ & 0,20 & $0,14 \pm 0,02$ & 70,0 & 10,3 \\
\hline & & 2,00 & $1,66 \pm 0,29$ & 83,0 & 10,8 \\
\hline & & 0,40 & $0,27 \pm 0,03$ & 67,5 & 5,10 \\
\hline & Querosene $^{\circ}$ & 2,00 & $0,90 \pm 0,20$ & 45,0 & 14,5 \\
\hline
\end{tabular}

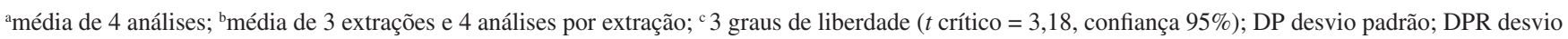
padrão relativo. 
Entretanto, os resultados obtidos para quantificação dos corantes SA-14 e SV-24 nas amostras de combustíveis avaliadas não foram satisfatórios, devido à sobreposição de picos interferentes presentes na matriz com os picos dos corantes avaliados nos comprimentos de onda de 640 e $490 \mathrm{~nm}$ seletivos para o SA-14 e SV-24, respectivamente. Ainda que ajustes no procedimento de extração desses analitos fossem realizados, os quais foram alternativamente desenvolvidos para melhorar a eficiência de extração, a complexidade da amostra como a de querosene, com diversidade de constituintes interferentes, constituiu-se em grande problema a ser contornado. Além disso, as diferenças de polaridade desses corantes também se constituíram em dificuldades adicionais no ajuste da eficiência do processo, uma vez que as interações com a fase estacionária foram decisivamente comprometidas, propiciando dificuldades em monitorar a eluição seletiva.

Sendo assim, a Tabela 3 apresenta apenas os resultados de recuperação empregando-se a técnica cromatográfica com detecção eletroquímica.

\section{CONCLUSÕES}

Os resultados obtidos revelam que a técnica de CLAE, acoplada ao detector eletroquímico, empregada na quantificação dos corantes SA-14 e SV-24 nas amostras de combustíveis apresentou desempenho bastante promissor, de modo que esta técnica pode ser uma alternativa na quantificação destes corantes nestas matrizes. Além disso, o método desenvolvido apresenta resultados analíticos satisfatórios, cujas informações são de grande relevância em estudos quantitativos envolvendo estas classes de compostos e pode contribuir efetivamente para o sucesso em análises desses e/ou outros tipos de corantes portadores de grupos azos e antraquinona em amostras de combustíveis. Considerando a complexidade da matriz, a separação em coluna de fase reversa (RP-C18) atendeu às necessidades específicas do estudo, com limite de quantificação suficiente para detecção desses corantes em amostras mais diluídas do que aqueles usualmente encontrados em trabalhos reportados na literatura. ${ }^{10-15}$

\section{AGRADECIMENTOS}

À FAPESP (processo 05/ 00382-4), CAPES e CNPq pelo apoio financeiro.

\section{REFERÊNCIAS}

1. Agência Nacional do Petróleo; http://www.anp.gov.br/doc/legislacao/ P2482000.pdf, acessada em Maio 2009.

2. European Refining \& Marketing; Fuels Refining \& Marketing in Europe and The Former Soviet Union, 2002, vol. 1, n. 10, http://www.processnmr.com/pdfs/erm-v1-10-02.pdf, acessada em Junho 2009.

3. Environmental Protection Agency (EPA); The Oil and Gas J. 1993, 91, 30.

4. The hydrocarbon oil (marking) regulations 2002; http://www.opsi.gov. uk/SI/si2002/20021773.htm, acessada em Junho 2009.

5. Orelup, R. B.; U. S. Patent 4735631,1988

6. Friswell, M. R.; Orelup, R. B.; U. S. Patent 5156653, 1992.

7. Guarantini, C. C. I.; Zanoni, M. V. B.; Quim. Nova 2000, 23, 71.

8. Zollinger, H.; Color Chemistry, $2^{\text {nd }}$ ed., V. C. H. Publishers: New York, 1991.

9. Abrahant, E. N.; Dyes and Their Intermediates, Edward Arnold Ltd.: London, 1977.

10. Barwick, V. J.; Ellison, S. L. R.; Rafferty, M. J. Q.; Gill, R. S., Accred. Qual. Assur. 2000, 5, 104.

11. Sundberg, L. N.; Andrasko, J.; Wistedt, I.; Kopp, I.; J. Foren. Sci. 1996 , 41,300 .

12. Henricsson, S.; Westerholm, R.; J. Chromatogr., A 1996, 723, 395.

13. Linsinger, T.; Koomen, G.; Emteborg, H.; Roebben, G.; Kramer, G.; Lamberty, A.; Energy \& Fuels 2004, 18, 1851.

14. Timkovich, R.; Dyes Pigm. 2000, 46, 69.

15. Leung, C. P.; Tam, S. Y. K.; Analyst 1985, 110, 883.

16. Trindade, M. A. G.; Ferreira V. S.; Zanoni, M. V. B.; Dyes Pigm. 2007, $74,566$.

17. Trindade, M. A. G.; Zanoni, M. V. B.; Electroanalysis 2007, 19, 1901.

18. Miller, J. C.; Miller, J. N.; Statistics for Analytical Chemistry, Ellis Horwood: New York, 1993. 